\title{
Balancing the brain: resting state networks and deep brain stimulation
}

\author{
Morten L. Kringelbach ${ }^{1,2,3}$, Alexander L. Green ${ }^{3}$ andTipu Z. Aziz ${ }^{2,3}$ \\ 1 Department of Psychiatry, Warneford Hospital, University of Oxford, Oxford, UK \\ 2 Centre for Functionally Integrative Neuroscience, University of Aarhus, Aarhus, Denmark \\ ${ }^{3}$ Nuffield Department of Surgery, John Radcliffe Hospital, Oxford, UK
}

\section{Edited by:}

Chiara Saviane, Scuola Internazionale

Superiore di Studi Avanzati, Italy

Reviewed by:

Hiroki Toda, The Tazuke Kofukai Medical

Research Institute Kitano Hospital,

Japan

Jens Kuhn, University of Cologne,

Germany

\section{${ }^{*}$ Correspondence:}

Morten L. Kringelbach, Department of

Psychiatry, Warneford Hospital,

University of Oxford, Oxford OX3 7JX

UK.

e-mail: morten.kringelbach@psych.

ox.ac.uk
Over the last three decades, large numbers of patients with otherwise treatment-resistant disorders have been helped by deep brain stimulation (DBS), yet a full scientific understanding of the underlying neural mechanisms is still missing. We have previously proposed that efficacious DBS works by restoring the balance of the brain's resting state networks. Here, we extend this proposal by reviewing how detailed investigations of the highly coherent functional and structural brain networks in health and disease (such as Parkinson's) have the potential not only to increase our understanding of fundamental brain function but of how best to modulate the balance. In particular, some of the newly identified hubs and connectors within and between resting state networks could become important new targets for DBS, including potentially in neuropsychiatric disorders. At the same time, it is of essence to consider the ethical implications of this perspective.

Keywords: resting state networks, oscillations, spontaneous activity, affective disorders, movement disorders

\section{INTRODUCTION}

Over the last couple of decades, deep brain stimulation (DBS) has shown remarkable clinical efficacy and safety in helping with otherwise treatment-resistant problems such as movement disorders and chronic pain (Kringelbach et al., 2007b; Deniau et al., 2010). The underlying principles and neural mechanisms of DBS are not yet fully understood but translational research has shown that DBS directly changes brain activity in a controlled manner (McIntyre and Hahn, 2010) and that, in principle, the resulting effects are reversible (Perlmutter and Mink, 2006). Many chronic brain disorders are linked to disturbances in finely balanced oscillatory brain networks, and we have previously proposed that an important principle by which DBS might work is to help restore the balance of resting state networks (Kringelbach et al., 2010). Thus the identification and understanding of the structural and functional architecture of these neural networks have the potential to direct novel targets and treatments with DBS.

The purpose of this perspective is to review the current stateof-the-art in characterizing the structural and functional architecture of the brain with a view to how this can best be modulated through DBS. We will focus on the analysis of the spontaneous brain activity that give rise to the intrinsic dynamics of the brain, which can be measured as spatially and temporally segregated networks (Deco et al., 2011). Some of this activity can be measured with functional neuroimaging as spontaneous fluctuations in blood oxygen level-dependent (BOLD) functional MRI (fMRI) signal. These intrinsic measures are stable across sessions and participants, and remarkably quick to acquire over only a matter of minutes (Greicius, 2008), which opens up for their use in even severely impaired patient groups. The functional activity is linked to structural brain connectivity which can now be measured in vivo, and which to some extent constrain the functional networks (Bullmore and Sporns, 2009). The resulting brain networks are remarkable stable in healthy individuals but have been shown to break down in various brain disorders. This in turn opens up for the discovery of the function of the hubs and connectors that are controlling the activity within and between brain networks. We argue that a better understanding of the detailed breakdown of the sub-components of the resting state networks in brain disorders opens for a principled way to discover novel targets with DBS.

\section{INTRINSIC NETWORK DYNAMICS}

Over the last few years the focus of modern neuroimaging has started to shift from the study of extrinsic to intrinsic brain activity (Biswal et al., 2010). This change has been brought about by the realization that while the vast majority of neuroimaging studies have been devoted to studying task-related changes in brain activity, the additional energy associated with this activity is remarkable low, often less than 5\% (Raichle and Mintun, 2006). Instead, the majority of brain energy consumption is devoted to intrinsic brain activity.

This intrinsic brain activity was mapped during the rest period in cognitive studies where researchers found a network of brain regions with remarkably high rates of change in metabolic markers such as cerebral blood flow, oxygen extraction and BOLD fMRI ( Lou et al., 1999). This network of brain regions was termed the Default Mode Network where the main regions in the network showed the largest deactivations during extrinsic cognitive tasks (Raichle and Mintun, 2006). While the network was initially thought to subserve internal modes of cognition such as representations of self (Buckner et al., 2008), this view is challenged by the persistence of the default mode network during light anesthesia in humans (Greicius et al., 2008) and monkeys (Vincent et al., 2006), as well as during early stages of sleep (Fukunaga et al., 2006). 
Other strands of research have focused on measuring the temporal correlation of spontaneous low-frequency BOLD signal fluctuations (Biswal et al., 1995). The measurement of these spontaneous fluctuations across various brain regions in the absence of an overt task has identified multiple functional resting state networks including the default mode network (Lowe et al., 1998; Greicius et al., 2003). Sophisticated independent component analyses of resting state patterns have identified at least seven networks which stay coherent over several minutes (Damoiseaux et al., 2006). Based on their brain components, these networks have been classified in (1) primary input-output networks (including sensorimotor, visual, auditory regions), (2) higher integrative networks (including attention, language, default mode, and executive regions; Beckmann et al., 2005), and (3) cortico-subcortical networks (including structures as the thalamus, basal ganglia, and cerebellum; Fox and Raichle, 2007). Interestingly, regions of the default mode network will remain tightly coherent but tend to show negative correlations with task-positive regions in the other networks.

The intrinsic activity of the human brain must be closely related to the large-scale anatomical connectivity between brain regions. Techniques such diffusion spectrum imaging and graph theory have revealed that the human brain exhibits a special kind of topology known as small-world architecture (Watts and Strogatz, 1998), which is characterized by high levels of local clustering among neighboring nodes (Hagmann et al., 2007; Bullmore and Sporns, 2009). Some nodes have higher connectivity in comparison with other nodes and are called hubs (He et al., 2009). The default mode network mostly consists of hubs, and in particular the precuneus and posterior cingulate cortex have been proposed to form the structural core (Hagmann et al., 2008). In general, structural and functional connectivity are linked, with the former predicting the latter (Honey et al., 2007). However, strong functional connectivity can exist between regions with no direct structural connection but that indirect connections and inter-regional distance to some extent can account for this (Honey et al., 2009).

This opens up the question of why these resting state networks exist in the first place. A long line of research has shown that the brain is primarily concerned with creating predictions optimizing input-output, which are then compared and updated accordingly (Friston, 2005). A potential explanation of the brain dynamics at rest has therefore been proposed to be linked to this constant state of exploration (Deco et al., 2011). The dynamics of the resting state networks could represent a metastable state; i.e., a state which persists for an extended period of time away from the natural equilibrium state. The brain is constantly exploring the potential functional network configurations, which over longer time windows will come to reflect the anatomical connectivity but over shorter time scales may be considerably more varied according to the impact of environmental demands. Computational models have shown how the important parameters in this process include local and global dynamics, noise, and signal transmission delay (Deco et al., 2009).

What has become clear is that neither the general neural dynamics, the structural connectivity, or the functional resting state networks are fully formed at birth but are being shaped during development (Fransson et al., 2007). As a result, important differences exist between infants, children, and adults (Gao et al., 2009; Supekar et al., 2010; Fan et al., 2011). The maturational processes must be driven foremost by extrinsic, environmental demands (Power et al., 2010) but also by intrinsic changes such as white matter maturation reshaping structural connectivity (Hagmann et al., 2010). It has been proposed that the epigenetic influences on shaping the neural dynamics of the resting state networks are at least as important as genetic factors, and can have a lasting impact on a number of important variables influencing quality of life, especially during the first 18 months (Parsons et al., 2010). One major variable of quality of life is overall hedonic tone, i.e., the likelihood of enjoying life and not suffering from anhedonia in mental disorders such as depression and anxiety. We have previously speculated that the default mode network may have an important role in shaping our overall well-being (Kringelbach and Berridge, 2009) (see Figure 1).

\section{BALANCING RESTING STATE NETWORKS IN DISEASE}

In general, resting state networks have been found to undergo significant, if sometimes only temporary changes in chronic brain disorders such as neuropsychiatric disorders (Greicius, 2008; Broyd et al., 2009). The causes of these perturbations are currently not well understood but it is clear that successful treatments somehow rebalance resting state networks. In disorders with no known pathology such as depression and anxiety, the subsequent rebalancing can occur spontaneously, or through carefully targeted interventions of either a cognitive nature (Teasdale et al., 2000) or even through more invasive methods such as DBS (Bewernick et al., 2010).

In pathological disorders with known pathologies like Parkinson's disease (PD) or chronic pain, spontaneous rebalancing is much less common and treatments often relies on heavily on understanding the system through the appropriate translational methods. In the case of PD, significant progress has been made through a number of animal models including the highly successful 1-methyl-4-phenyl-1,2,3,6-tetrahydropyridine (MPTP) model in higher primates (Langston et al., 1983). This model has helped identify a number of efficacious DBS targets such as the subthalamic nucleus (STN; Bergman et al., 1990; Aziz et al., 1991).

In PD, the loss of dopaminergic cells means that the basic oscillations between cortex and subcortical regions become unbalanced. Human studies have found there are strong increases in beta $(15-30 \mathrm{~Hz})$ oscillatory activity in the STN when the patients are without dopaminergic medication, while therapeutic effective STN stimulation of larger than $70 \mathrm{~Hz}$ has the effect of suppressing this noisy activity in the basal ganglia (Brown et al., 2004).

Careful animal and human experimentation have thus given a better understanding of some of the fundamental principles of the breakdown in PD of how brain regions oscillate and communicate (Schnitzler and Gross, 2005). DBS has in turn brought some relief for over 60,000 PD patients since the early 1990s.

On the basis of this experimentation, some conclusions can be drawn about the neural and systems level mechanisms of action of DBS. The effects of DBS are closely linked to at least three factors: (1) the stimulation parameters (including frequency, amplitude, pulse width, and duration); (2) the intrinsic physiological properties of the neural tissue which may change with disease state; and (3) the interactions between the electrode and the geometric configuration of the surrounding neural tissue and specific anatomy 
of the targeted region (Kringelbach et al., 2007b). The evidence clearly shows that DBS affects multiple neural elements; foremost myelinated axons - and to a lesser degree cell bodies.

The fundamental mechanism of DBS is through stimulationinduced modulation of the activity of larger brain networks (Montgomery and Baker, 2000; Vitek, 2002; McIntyre et al., 2004; Kringelbach et al., 2007b; McIntyre and Hahn, 2010). This has been confirmed by optogenetic experiments in rodents which show that the therapeutic effects within the STN can be accounted for by direct selective stimulation of afferent axons projecting to this region (Gradinaru et al., 2009).

Despite the remarkable successes in treating PD with DBS, it is not clear at this point if the existing targets and treatments are the most efficacious. The oscillatory activity clearly reflects a variety of motor and cognitive-emotional processes but it is not clear how disease severity or extrinsic task demands affect the neural dynamics of PD (Vardy et al., 2010). Neuroimaging studies have, however, shown that the default mode network exhibits specific changes in PD (van Eimeren et al., 2009; Delaveau et al., 2010). Significant functional differences were found in the posterior cingulate cortex and precuneus, and connectivity analysis showed that the medial prefrontal cortex and rostral ventromedial caudate nucleus were functionally disconnected in PD. Some of these changes can be restored with administration of levodopa (Delaveau et al., 2010). Yet, so far DBS has not been used in any of the affected hubs of the default mode network in PD.

The large body of PD research mapping the underlying mechanisms of DBS has not yet been matched by a similar body of evidence for the emerging DBS treatment of neuropsychiatric disorders (Kopell and Greenberg, 2008). Interestingly, however, it should be noted that many of the brain structures involved in movement disorders are also implicated in affective disorders. This is for example demonstrated by how severe depression can be reversibly induced by DBS for PD (Bejjani et al., 1999; Temel et al., 2006).

From the present perspective, these transient changes are of considerable interest taken together with the demonstrated altered activity in affective disorders for many of the main hubs and connectors such as the subgenual cingulate cortex, orbitofrontal cortex, ventral pallidum, and nucleus accumbens (Giacobbe et al., 2009; Bewernick et al., 2010). These reward-related brain regions are known to modulate hedonic state and as such could be responsible for the debilitating anhedonia found in affective disorders (Kringelbach, 2005; Berridge and Kringelbach, 2008). One possible hypothesis is thus that DBS for affective disorders could work by modulating the hedonic circuitries in order to alleviate anhedonia (Kringelbach and Berridge, 2009; Kringelbach et al., 2010).

\section{NOVEL RESEARCH AVENUES}

The future of direct brain interventions will rely on having a much better understanding of the fundamental nature of intricate dynamics of the resting state networks. Most of the current evidence has come from neuroimaging techniques such as BOLD fMRI and positron emission tomography which are only indirect, correlational measures of neural activity. The dynamic nature of the short and long-term brain activity means that we will need a far more detailed understanding of underlying signals, including computational modeling (Deco et al., 2011). More temporally suitable neuroimaging methods such as magnetoencephalography (MEG) are starting to address these shortcomings (Hansen et al., 2010).

In fact, combining MEG and DBS may offer new insights into the fine-grained temporal neural dynamics of aberrant brain states, while at the same time providing novel insights into the fundamental principles as first demonstrated in 2006 (Kringelbach et al., 2007a) (see Figure 1C). The technical challenges of using an invasive technique with a highly sensitive method are significant but not insurmountable. One subsequent study used simultaneous MEG and local field potential (LFP) recordings from the DBS electrode to demonstrate that an image analysis method called beamforming is capable of suppressing the high-amplitude artifacts caused by the DBS wire and electrode and extracting artifact-free virtual electrode time-series (Litvak et al., 2010). Another study using DBS, LFP, and MEG found frequency-specific functional connectivity between basal ganglia and cortex in $\mathrm{PD}$, suggesting

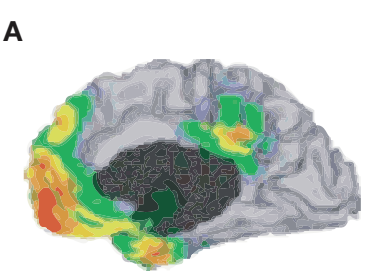

Normal resting state
B

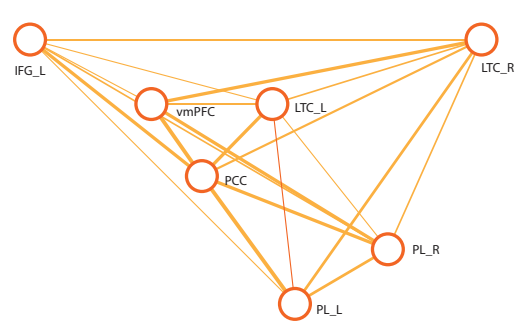

C

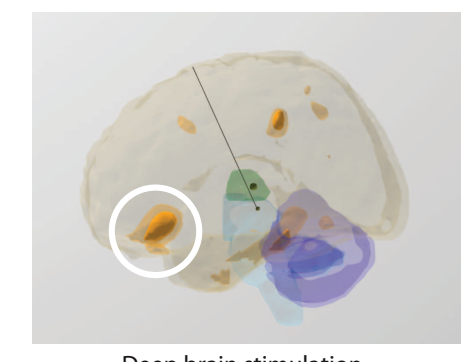

Deep brain stimulation

FIGURE 1 | (A) The brain's default mode network is a steady state circuit of the brain where activity increases during rest (Gusnard and Raichle, 2001). The coloring indicates brain areas with significant levels of blood flow. (B) In the normal brain, this brain network can be described in terms of functional connectivity graphs. More strongly connected regions (indicated by heavier orange lines) are clustered near each other while weakly correlated regions are placed further away with the line width proportional to the connection strength (Gao et al., 2009). (C) Deep brain stimulation causally alters brain activity and future challenges include how to best restore this default network in malignant brain disorders. We made a tentative start by using MEG to investigate how a deep brain stimulation electrode implanted in the PVG/PAG can help a wider network alleviate the suffering of chronic pain (Kringelbach et al., 2007a). The three-dimensional rendering shows the significant increases in activity in shades of orange, e.g., in regions such as the mid-anterior orbitofrontal cortex (white circle), while the other colors represent landmark brain structures: thalamus (green), cerebellum (blue), and brainstem (light blue). IFG_L, IFG_R left and right inferior frontal gyrus; LTC_L, LTC_R, left and right lateral temporal cortex; PCC, posterior cingulate cortex/ retrosplenial; $\mathrm{PL} \_\mathrm{L}, \mathrm{PL} \_\mathrm{R}$, left and right parietal lobes; vmPFC, ventromedial prefrontal cortex. 
that simultaneous inter-regional interactions may be segregated in the frequency domain (Hirschmann et al., 2010). Specifically, coherent activity was found in the low and high beta range in the ipsilateral sensorimotor and the premotor cortex, while coherence in the alpha range $(7-12 \mathrm{~Hz})$ was observed at various locations in the ipsilateral temporal lobe.

We combined DBS and MEG to obtain important new information about the underlying neural dynamics in a rare case of whole-body chronic pain (Mohseni et al., 2010). We stimulated one of the main hubs of the brain, the anterior cingulate cortex, and found the stimulation-specific alleviation of whole-body pain was modulating brain activity in a wide spread network of regions including the pre-supplementary motor area, brainstem periaqueductal gray, rostral anterior cingulate cortex, and medial prefrontal areas. Given the lack of good animal models for chronic pain, this information could be important for guiding future treatments. It could also be pertinent to our general understanding of the role of the anterior cingulate cortex in refractory depression, pain, and obsessive-compulsive disorder, and in particular why bilateral anterior cingulotomies have been found to have some beneficial effects (Steele et al., 2008).

\section{DISCUSSION}

We are only beginning to understand the neural dynamics underlying the intrinsic structural and functional brain activity (Biswal et al., 2010). It is, however, clear that specific modulation of these

\section{REFERENCES}

Aziz, T. Z., Peggs, D., Sambrook, M. A., and Crossman, A. R. (1991). Lesion of the subthalamic nucleus for the alleviation of 1-methyl-4-phenyl-1,2,3,6tetrahydropyridine (MPTP)-induced parkinsonism in the primate. Mov. Disord. 6, 288-292.

Beckmann, C. F., DeLuca, M., Devlin, J. T., and Smith, S.M. (2005). Investigations into resting-state connectivity using independent component analysis. Philos. Trans. R. Soc. Lond. B Biol. Sci. 360, 1001-1013.

Bejjani, B. P., Damier, P., Arnulf, I., Thivard, L., Bonnet, A. M., Dormont, D., Cornu, P., Pidoux, B., Samson, Y., and Agid, Y. (1999). Transient acute depression induced by high-frequency deep-brain stimulation. N. Engl. J. Med. 340, 1476-1480.

Bergman, H., Wichmann, T., and DeLong, M. R. (1990). Reversal of experimental parkinsonism by lesions of the subthalamic nucleus. Science 249, 1436-1438.

Berridge, K. C., and Kringelbach, M. L. (2008). Affective neuroscience of pleasure: reward in humans and animals. Psychopharmacology (Berl.) 199, 457-480.

Bewernick, B. H., Hurlemann, R., Matusch, A., Kayser, S., Grubert, C., Hadrysiewicz, B., Axmacher, N., Lemke, M., Cooper-Mahkorn, D., Cohen, M. X., Brockmann, H., Lenartz, D., Sturm, V., and Schlaepfer,
T.E. (2010). Nucleus accumbens deep resistant depression. Biol. Psychiatry 67, 110-116.

Biswal, B, Yetkin, F. Z., Haughton, V. M., and Hyde, J. S. (1995). Functional connectivity in the motor cortex of resting human brain using echo-planar MRI. Magn. Reson. Med. 34, 537-541.

Biswal, B. B., Mennes, M., Zuo, X. N., Gohel, S., Kelly, C., Smith, S. M., Beckmann, C. F., Adelstein, J. S., Buckner, R. L., Colcombe, S., Dogonowski, A. M., Ernst, M., Fair, D., Hampson, M., Hoptman, M. J., Hyde, J. S., Kiviniemi, V. J., Kotter, R., Li, S. J., Lin, C. P., Lowe, M. J., Mackay, C., Madden, D. J., Madsen, K. H., Margulies, D. S., Mayberg, H. S., McMahon, K., Monk, C. S., Mostofsky, S. H., Nagel, B. J., Pekar, J. J., Peltier, S. J., Petersen, S. E., Riedl, V., Rombouts, S.A., Rypma, B., Schlaggar, B. L., Schmidt, S., Seidler, R. D., Siegle, G. J., Sorg, C., Teng, G. J., Veijola, J., Villringer, A., Walter, M., Wang, L., Weng, X. C., Whitfield-Gabrieli, S., Williamson, P., Windischberger, C., Zang, Y. F., Zhang, H. Y., Castellanos, F. X., and Milham, M. P. (2010). Toward discovery science of human brain function. Proc. Natl. Acad. Sci. U.S.A. 107, 4734-4739.

Brown, P., Mazzone, P., Oliviero, A., Altibrandi, M. G., Pilato, F., Tonali, P. brain stimulation decreases ratings of depression and anxiety in treatment-

resting state networks in disease could bring significant benefits for future treatments of chronic brain disorders. The demonstrated clinical efficacy and safety of DBS means that this technique is an important tool for rebalancing the resting state networks (Kringelbach et al., 2010).

In this perspective article, we have reviewed the current evidence for DBS as a tool for modulation of the activity of the highly coherent functional and structural brain networks in health and disease. In particular, we have focused on how some of the hubs and connectors within and between resting state networks of the brain could become important new stimulation targets, including potentially in neuropsychiatric disorders.

Overall, DBS remains an important tool both for alleviating human suffering and for obtaining novel insights into the nature of fundamental brain function. Combining DBS with a better understanding of the intrinsic activity of the brain may come to serve as an important tool for rebalancing resting state network activity in chronic brain disorders. Yet, this promising avenue for discovering novel DBS targets must be guided by careful ethical considerations (Kringelbach and Aziz, 2009; Schlaepfer and Fins, 2010).

\section{ACKNOWLEDGMENTS}

The authors were funded by TrygFonden Charitable Foundation, Medical Research Council, Norman Collisson Foundation, and Charles Wolfson Charitable Trust.

A., and Di Lazzaro, V. (2004). Effects of stimulation of the subthalamic area on oscillatory pallidal activity in Parkinson's disease. Exp. Neurol. 188, 480-490.

Broyd, S. J., Demanuele, C., Debener, S. Helps, S. K., James, C. J., and SonugaBarke, E. J. (2009). Default-mode brain dysfunction in mental disorders: a systematic review. Neurosci. Biobehav. Rev. 33, 279-296.

Buckner, R. L., Andrews-Hanna, J. R., and Schacter, D. L. (2008). The brain's default network: anatomy, function, and relevance to disease. Ann. N. Y. Acad. Sci. 1124, 1-38.

Bullmore, E., and Sporns, O. (2009). Complex brain networks: graph theoretical analysis of structural and functional systems. Nat. Rev. Neurosci. 10, 186-198.

Damoiseaux, J. S., Rombouts, S. A. R. B., Barkhof, F., Scheltens, P., Stam, C. J., Smith, S. M., and Beckmann, C. F. (2006). Consistent resting-state networks across healthy subjects. Proc. Natl. Acad. Sci. U.S.A. 103 13848-13853.

Deco, G., Jirsa, V., McIntosh, A. R., Sporns, O., and Kotter, R. (2009). Key role of coupling, delay, and noise in resting brain fluctuations. Proc. Natl. Acad. Sci. U.S.A. 106, 10302-10307.

Deco, G., Jirsa, V. K., and McIntosh, A. R. (2011). Emerging concepts for the dynamical organization of resting state activity in the brain. Nat. Rev. Neurosci. 12, 43-56.

Delaveau, P., Salgado-Pineda, P., Fossati, P., Witjas, T., Azulay, J. P., and Blin, O. (2010). Dopaminergic modulation of the default mode network in Parkinson's disease. Eur. Neuropsychopharmacol. 20, 784-792.

Deniau, J. M., Degos, B., Bosch, C., and Maurice, N. (2010). Deep brain stimulation mechanisms: beyond the concept of local functional inhibition. Eur. J. Neurosci. 32, 1080-1091.

Fan, Y., Shi, F., Smith, J. K., Lin, W. Gilmore, J. H., and Shen, D. (2011). Brain anatomical networks in early human brain development. Neuroimage 54, 1862-1871.

Fox, M. D., and Raichle, M. E. . (2007). Spontaneous fluctuations in brain activity observed with functional magnetic resonance imaging. Nat. Rev. Neurosci. 8, 700-711.

Fransson, P., Skiold, B., Horsch, S., Nordell, A., Blennow, M., Lagercrantz, H., and Aden, U. (2007). Resting-state networks in the infant brain. Proc. Natl. Acad. Sci. U.S.A. 104, 15531-15536.

Friston, K. (2005). A theory of cortical responses. Philos. Trans. R. Soc. Lond. B Biol. Sci. 360, 815-836.

Fukunaga, M, Horovitz, S. G., van Gelderen, P., de Zwart, J. A., Jansma, J. M., Ikonomidou, V. N., Chu, R., Deckers, R. H., Leopold, D. A., and Duyn, J. H. (2006). Large amplitude, 
spatially correlated fluctuations in BOLD fMRI signals during extended rest and early sleep stages. Magn. Reson. Imaging 24, 979-992.

Gao, W., Zhu, H., Giovanello, K. S., Smith, J. K., Shen, D., Gilmore, J. H., and Lin, W. (2009). Evidence on the emergence of the brain's default network from 2-week-old to 2-year-old healthy pediatric subjects. Proc. Natl. Acad. Sci. U.S.A. 106, 6790-6795.

Giacobbe, P., Mayberg, H. S., and Lozano, A. M. (2009). Treatment resistant depression as a failure of brain homeostatic mechanisms: implications for deep brain stimulation. Exp. Neurol. 219, 44-52.

Gradinaru, V., Mogri, M., Thompson, K. R., Henderson, J. M., and Deisseroth, K. (2009). Optical deconstruction of Parkinsonian neural circuitry. Science 3247, 354-359.

Greicius, M. (2008). Resting-state functional connectivity in neuropsychiatric disorders. Curr. Opin. Neurol. 21, 424-430.

Greicius, M. D., Kiviniemi, V., Tervonen, O., Vainionpää, V., Alahuhta, S., Reiss, A. L., and Menon, V. (2008). Persistent default-mode network connectivity during light sedation. Hum. Brain Mapp. 29, 839-847.

Greicius, M. D., Krasnow, B., Reiss, A. L., and Menon, V. (2003). Functional connectivity in the resting brain: a network analysis of the default mode hypothesis. Proc. Natl. Acad. Sci. U.S.A. 100, 253-258.

Gusnard, D. A., and Raichle, M. E. (2001). Searching for a baseline: functional imaging and the resting human brain. Nat. Rev. Neurosci. 2, 685-694.

Hagmann, P., Cammoun, L., Gigandet, X., Meuli, R., Honey, C. J., Wedeen, V. J., and Sporns, O. (2008). Mapping the structural core of human cerebral cortex. PLoS Biol. 6, e159. doi: 10.1371/ journal.pbio.0060159

Hagmann, P., Kurant, M., Gigandet, X., Thiran, P., Wedeen, V.J., Meuli, R., and Thiran, J. P. (2007). Mapping human whole-brain structural networks with diffusion MRI. PLoS ONE2, e597.doi: 10.1371/journal.pone.0000597

Hagmann, P., Sporns, O., Madan, N., Cammoun, L., Pienaar, R., Wedeen, V. J., Meuli, R., Thiran, J. P., and Grant, P. E. (2010). White matter maturation reshapes structural connectivity in the late developing human brain. Proc. Natl. Acad. Sci. U.S.A. 107, 19067-19072.

Hansen, P. C., Kringelbach, M. L., and Salmelin, R. (2010). MEG. An Introduction to Methods. Oxford: Oxford University Press.

He, Y., Wang, J., Wang, L., Chen, Z. J., Yan, C., Yang, H., Tang, H., Zhu, C., Gong, Q., Zang, Y., and Evans, A. C. (2009).
Uncovering intrinsic modular organization of spontaneous brain activity in humans. PLoS ONE 4, e5226. doi: 10.1371/journal.pone.0005226

Hirschmann, J., Ozkurt, T. E., Butz, M., Homburger, M., Elben, S., Hartmann, C. J., Vesper, J., Wojtecki, L., and Schnitzler,A. (2010).Distinct oscillatory STN-cortical loops revealed by simultaneous MEG and local field potential recordings in patients with Parkinson's disease. Neuroimage 55, 1159-1168.

Honey, C. J., Kotter, R., Breakspear, M., and Sporns, O. (2007). Network structure of cerebral cortex shapes functional connectivity on multiple time scales. Proc. Natl. Acad. Sci. U.S.A. 104, 10240-10245.

Honey, C. J., Sporns, O., Cammoun, L., Gigandet, X., Thiran, J. P., Meuli, R., and Hagmann, P. (2009). Predicting human resting-state functional connectivity from structural connectivity. Proc. Natl. Acad. Sci. U.S.A. 106, 2035-2040.

Kopell, B. H., and Greenberg, B. D. (2008). Anatomy and physiology of the basal ganglia: implications for DBS in psychiatry. Neurosci. Biobehav. Rev. 32, 408-422.

Kringelbach, M. L. (2005). The orbitofrontal cortex: linking reward to hedonic experience. Nat. Rev. Neurosci. 6, 691-702.

Kringelbach, M. L., and Aziz, T.Z. (2009). Deep brain stimulation: avoiding the errors of psychosurgery. JAMA 301, 1705-1707.

Kringelbach, M. L., and Berridge, K. C. (2009). Towards a functional neuroanatomy of pleasure and happiness. Trends Cogn. Sci. (Regul. Ed.) 13, 479-487.

Kringelbach, M. L., Green, A. L., Owen, S. L. F., Schweder, P. M., and Aziz, T. Z. (2010). Sing the mind electric: principles of deep brain stimulation. Eur. J. Neurosci. 32, 1070-1079.

Kringelbach, M. L., Jenkinson, N., Green, A. L., Owen, S. L. F., Hansen, P. C., Cornelissen, P. L., Holliday, I. E., Stein, J., and Aziz, T. Z. (2007a). Deep brain stimulation for chronic pain investigated with magnetoencephalography. Neuroreport 18, 223-228.

Kringelbach, M. L., Jenkinson, N., Owen, S. L. F., and Aziz, T. Z. (2007b). Translational principles of deep brain stimulation. Nat. Rev. Neurosci. 8, 623-635.

Langston, J. W., Ballard, P., Tetrud, J. W., and Irwin, I. (1983). Chronic Parkinsonism in humans due to a product of meperidine-analog synthesis. Science 219, 979-980.

Litvak, V., Eusebio, A., Jha,A., Oostenveld, R., Barnes, G. R., Penny, W. D., Zrinzo, L., Hariz, M. I., Limousin, P., Friston, K. J., and Brown, P. (2010). Optimized beamforming for simultaneous MEG and intracranial local field potential recordings in deep brain stimulation patients. Neuroimage 50, 1578-1588.

Lou, H. C., Kjaer, T. W., Friberg, L. Wildschiodtz, G., Holm, S., and Nowak, M. (1999). A ${ }^{15} \mathrm{O}-\mathrm{H}_{2} \mathrm{O}$ PET study of meditation and the resting state of normal consciousness. Hum. Brain Mapp. 7, 98-105.

Lowe, M. J., Mock, B. J., and Sorenson, J. A. (1998). Functional connectivity in single and multislice echoplanar imaging using resting-state fluctuations. Neuroimage 7, 119-132.

McIntyre, C. C., and Hahn, P. J. (2010). Network perspectives on the mechanisms of deep brain stimulation. Neurobiol. Dis. 38, 329-337.

McIntyre, C. C., Savasta, M., Kerkerian-Le Goff, L., and Vitek, J. L. (2004). Uncovering the mechanism(s) of action of deep brain stimulation: activation, inhibition, or both. Clin Neurophysiol. 115, 1239-1248.

Mohseni, H. R., Kringelbach, M. L., Probert Smith, P., Parsons, C. E., Young, K. S., Hyam, J. A., Schweder, P. M., Stein, J. F., Green, A. L., and Aziz, T. Z. (2010). "Application of a nullbeamformer to source localisation in MEG data of deep brain stimulation," in 2010 Annual International Conference of the IEEE Engineering in Medicine and Biology Society, EMBC'10, Buenos Aires, 4120-4123.

Montgomery, E. B. Jr., and Baker, K. B. (2000). Mechanisms of deep brain stimulation and future technical developments. Neurol. Res. 22, 259-266.

Parsons, C. E., Young, K. S., Murray, L., Stein, A., and Kringelbach, M. L. (2010). The functional neuroanatomy of the evolving parent-infant relationship. Prog. Neurobiol. 91, 220-241.

Perlmutter, J. S., and Mink, J. W. (2006). Deep brain stimulation. Annu. Rev. Neurosci. 29, 229-257.

Power, J. D., Fair, D. A., Schlaggar, B. L., and Petersen, S. E. (2010). The development of human functional brain networks. Neuron 67, 735-748.

Raichle, M. E., and Mintun, M. A. (2006) Brain work and brain imaging. Annu. Rev. Neurosci. 29, 449-476.

Schlaepfer, T. E., and Fins, J. J. (2010). Deep brain stimulation and the neuroethics of responsible publishing. JAMA 303, 775-776.

Schnitzler, A., and Gross, J. (2005) Normal and pathological oscillatory communication in the brain. Nat. Rev. Neurosci. 6, 285-296.

Steele, J. D., Christmas, D., Eljamel, M. S., and Matthews, K. (2008). Anterior cingulotomy for major depression: clinical outcome and relationship to lesion characteristics. Biol. Psychiatry 63, 670-677.
Supekar, K., Uddin, L. Q., Prater, K., Amin, H., Greicius, M. D., and Menon, V. (2010). Development of functional and structural connectivity within the default mode network in young children. Neuroimage 52, 290-301.

Teasdale, J. D., Segal, Z. V., Williams, J. M., Ridgeway, V. A., Soulsby, J. M., and Lau, M. A. (2000). Prevention of relapse/recurrence in major depression by mindfulness-based cognitive therapy. J. Consult. Clin. Psychol. 68, 615-623.

Temel, Y., Kessels, A., Tan, S., Topdag, A., Boon, P., and Visser-Vandewalle, V. (2006). Behavioural changes after bilateral subthalamic stimulation in advanced Parkinson disease: a systematic review. Parkinsonism Relat. Disord. 12, 265-272.

van Eimeren, T., Monchi, O., Ballanger, B., and Strafella, A. P. (2009). Dysfunction of the default mode network in Parkinson disease: a functional magnetic resonance imaging study. Arch. Neurol. 66, 877-883.

Vardy, A. N., van Wegen, E. E., Kwakkel, G., Berendse, H. W., Beek, P. J., and Daffertshofer, A. (2010). Slowing of M1 activity in Parkinson's disease during rest and movement - an MEG study. Clin. Neurophysiol. 122, 789-795.

Vincent, J. L., Snyder, A. Z., Fox, M. D., Shannon, B. J., Andrews, J. R., Raichle, M. E., and Buckner, R. L. (2006). Coherent spontaneous activity identifies a hippocampal-parietal memory network. J. Neurophysiol. 96, 3517-3531.

Vitek, J. L. (2002). Mechanisms of deep brain stimulation: excitation or inhibition. Mov. Disord. 17, S69-S72.

Watts, D. J., and Strogatz, S. H. (1998). Collective dynamics of 'small-world' networks. Nature 393, 440-442.

Conflict of Interest Statement: The authors declare that the research was conducted in the absence of any commercial or financial relationships that could be construed as a potential conflict of interest.

Received: 01 January 2011; accepted: 18 April 2011; published online: 02 May 2011. Citation: Kringelbach ML, Green $A L$ and AzizTZ (2011) Balancing the brain: resting state networks and deep brain stimulation. Front. Integr. Neurosci. 5:8. doi: 10.3389/ fnint.2011.00008

Copyright $\odot 2011$ Kringelbach, Green and Aziz. This is an open-access article subject to a non-exclusive license between the authors and Frontiers Media SA, which permits use, distribution and reproduction in other forums, provided the original authors and source are credited and other Frontiers conditions are complied with. 